\title{
QUEENS COLLEGE RADIOCARBON MEASUREMENTS II
}

\author{
R PARDI
}

Radiocarbon Laboratory, Queens College, City University of New York Flushing, New York 11367

This list contains all analysis completed since the first list ( R, 1975, v 18, p 205).

Synthesis and counting are essentially the same as before except that the computer programs now employed reject sample counting intervals that fall $>2.5 \sigma$ from the mean.

Samples submitted for analysis are reviewed by a Committee consisting of W DeBoer, P Tolstoy, Anthropology, L Marcus, Biology, W S Newman, Earth and Environmental Sciences, and R Pardi, Radiocarbon Laboratory.

All results are based on the conventional half-life for ${ }^{14} \mathrm{C}, i e, 5568 \pm$ 30 years. Results are reported in years before 1950. Errors are $1 \sigma$, based on the combined statistical counting error of the sample, background and standard. ${ }^{13} \mathrm{C} /{ }^{12} \mathrm{C}$ measurements and corrections have not been made for these samples.

\section{ACKNOWLEDGMENTS}

Sue Coughlin, Don Haarmann, and Linda Kaplan assisted in sample preparation and compiling this list. The continued support and encouragement of David H Speidel is greatly appreciated. I am indebted to L Marcus for his assistance with the computer programming.

I. GEOLOGIC SAMPLES

\section{QC-122. USAF Montauk 1}

Peaty clay from modern bluff, US Air Force Stat, Montauk Point, New York $\left(41^{\circ} 03^{\prime} \mathrm{N}, 71^{\circ} 52^{\prime} \mathrm{W}\right)$, alt ca $\mathrm{lm} \mathrm{MHW}$. Coll and subm by W S Newman, Queens Coll, CUNY, Flushing, New York. Date on acidwashed organic fragments of $>40$ mesh. Comment (WSN): proglacial (?) lacustrine deposit above Montauk till and near base of overlying stratified drift. Top of sec includes some till lenses believed to be Late Wisconsin in age. Previous date on $\log$ from nearby locality, RL-318: $38,800+5600$ / -3200 .

\section{Great Bear Swamp series}

Peat from Great Bear swamp, central Albany Co, New York $\left(42^{\circ}\right.$ $28^{\prime} \mathrm{N}, 74^{\circ} 03^{\prime} \mathrm{W}$ ) alt ca $366 \mathrm{~m}$ ( $c f$, Connally and Sirkin, 1971). Coll and subm by R J Dineen, New York State Geol Survey, Albany, New York. $\mathrm{HCl}$ pretreatment only.

General Comment (RJD): upland swamp, dammed by glacial debris.

QC-148. 1,150 to $170 \mathrm{~cm}$ $4000 \pm 100$

Comment (RJD): dates middle or early Pine Zone development in this area. Sample was pooled from 2 cores at layer 150 to $170 \mathrm{~cm}$. Possible contamination by root penetration. 
QC-149. 2, 316 to $340 \mathrm{~cm}$

$11,590 \pm 265$

Sample diluted with "dead" benzene. Comment (RJD): dates postglacial lake that occupied Great Bear Swamp depression after ice retreated $\mathrm{N}$ to lowlands. Sample pooled from 2 cores at layer 326 to $340 \mathrm{~cm}$. Possible contamination by root penetration.

\section{QC-153. Norfolk 5d}

$11,230 \pm 200$

Shells of small pelycepod from glacial Champlain Sea, St Lawrence Lowland, Winnowed Till Sec, NE of Norfolk, New York $\left(44^{\circ} 51^{\prime} \mathrm{N}, 74^{\circ}\right.$ $52^{\prime} \mathrm{W}$ ), alt $88 \mathrm{~m}$, depth $1.5 \mathrm{~m}$. Coll and subm by D R Coates, Dept Geol Sci, SUNY at Binghamton, New York. Comment (DRC): from beach and strandline-type deposits with sandy matrix. Previous dates on Champlain Sea samples from Brock: 10,340 $\pm 130,11,000 \pm 150$ and 12,000 \pm 200 .

\section{QC-154. Gay Head}

$23,900 \pm 450$

Wood-charcoal from Gay Head-central S-side, mouth of Devil's den, Martha's Vineyard, Massachusetts $\left(41^{\circ} 21^{\prime} \mathrm{N}, 70^{\circ} 51^{\prime} \mathrm{W}\right)$. Coll and subm by C Kaye, US Geol Survey, 80 Broad St, Boston, Massachusetts. Comments (CK): lignitized wood or fusain from shear plane in Cretaceous white clayey sand. (RRP): previous date on material from same locality coll by RRP, GX-3000: 23,900 +2200/-1700.

\section{QC-159. Cape Cod 1}

1) $>44,000$

2) $>43,000$

Wood from just NE of Highland Light, N Thuro quad, Massachusetts $\left(40^{\circ} 2^{\prime} 30^{\prime \prime} \mathrm{N}, 70^{\circ} 3^{\prime} 40^{\prime \prime} \mathrm{W}\right)$, approx alt $11 \mathrm{~m}$ ASL, depth ca $26 \mathrm{~m}$ below surface. Coll and subm by J H Hartshorn, Dept Geol/Geog, Univ Massachusetts, Amherst, Massachusetts. Comments: sample treated with $5 \% \mathrm{NaOH}$. (JHH): wood is not in situ. Estimated age at least 35,000 yr $\mathrm{BP}$, based on previous dates in area and stratigraphic considerations.

\section{QC-162. Mal 28-70}

$3400 \pm 125$

Shell from esker resting on till, Malaspina glacier $\left(59^{\circ} 47^{\prime} \mathrm{N}, 140^{\circ}\right.$ $\left.10^{\prime} \mathrm{W}\right)$, ca $23 \mathrm{~m}$ ASL. Comment (JHH): in 1951 the esker was just beginning to emerge from beneath ice. Shells date from ice recession when sea was far $\mathbf{N}$ of present limits. Readvance incorporated shells first in till and then in meltwater stream gravels.

\section{Deep sea core series}

Carbonate fraction of deep sea cores V30-97 $\left(41^{\circ} 00^{\prime} \mathrm{N}, 32^{\circ} 55.5^{\prime} \mathrm{W}\right)$, V22-82 $\left(00^{\circ} 32.5^{\prime} \mathrm{S}, 17^{\circ} 16^{\prime} \mathrm{W}\right)$, subm by A McIntyre, Lamont-Doherty Geol Observatory, Palisades, New York. All samples were diluted with "dead" benzene.

$$
\begin{aligned}
& \text { QC-198. V30-97, } 63.5 \text { to } 71.5 \mathrm{~cm} \\
& \text { Ca 53\% } \mathrm{CaCO}_{3} .
\end{aligned}
$$$$
16,360 \pm 220
$$

QC-215. V22-182, 85 to $89 \mathrm{~cm}$

$21,000 \pm 770$

$\mathrm{Ca} 95 \% \mathrm{CaCO}_{3}$. 
QC-216. V22-182, 45 to $49 \mathrm{~cm}$

Ca $82 \% \mathrm{CaCO}_{3}$.

\section{Pt Peron series}

QC-191.A Pt Peron

$4115 \pm 105$

QC-191.C Pt Peron

$3910 \pm 125$

Mixed gastropods and fragments from Pt Peron, W Australia $\left(32^{\circ} \mathrm{S}\right.$, $116^{\circ} \mathrm{E}$ ), alt $4.5 \mathrm{~m}$ above LWL. Coll and subm by R Fairbridge, Columbia Univ, New York. A: whole shell and fragments of whelk and cone gastropods, C: 3 large horn gastropods.

\section{Leit's Beach, Victoria series}

Shells from Leit's Beach, Victoria $\left(38^{\circ} \mathrm{S}, 148^{\circ} \mathrm{E}\right)$. Coll by R Ward and R Fairbridge. Subm by R Fairbridge.

QC-192. Seacombe 2

Modern beach shells.

$100.4 \pm 1.0 \%$

QC-193. Leit's Beach 3

$475 \pm 90$

Pelycepod shells from midden 4.5 to $6 \mathrm{~m}$ above beach.

QC-194. Dune Causeway 4

$1750 \pm 130$

Shell midden from inner margin of fore dunes. Same pelycepod species as QC-192 \& -193.

\section{QC-195. Lagoon 6}

$5980 \pm 165$

Snail and clam shells from outcrop at high tide level.

\section{Champlain Sea series}

Shells from glacial Champlain Sea, NE New York State. Coll and subm by T M Cronin, Mus Comparative Zool, Harvard Univ, Cambridge, Massachusetts.

General Comment (TMC): both species could inhabit brackish water down to 5ppt.

\section{QC-199. T C 92}

$10,300 \pm 180$

Mya arenaria shell, Beekmantown, New York $\left(44^{\circ} 47^{\prime} \mathrm{N}, 73^{\circ} 6^{\prime} \mathrm{W}\right)$, alt $40 \mathrm{~m}$ ASL. Comment (TMC): date indicates deposition near end of Late Pleistocene from sea (see Hillaire-Marcel, 1974, for a list of Canadian dates). Youngest date yet recorded for New York State (cf, W-1109: 10,560 \pm 350 , Ives et al, 1964) and 2nd youngest date from Champlain Valley (cf I-4695: $9620 \pm 350$, from West Bridgport, Vermont, Wasner, 1972). Most significantly, date demonstrates existence of Mya arenaria phase (Elson, 1969) of the sea of New York and also that Mya phase deposits are correlative, based on alt ASL and fauna, with deposits in Quebec that yield similar faunas and late Champlain Sea ages ( $c f$, Elson, 1962, GrN2032: $10,450 \pm 80, \mathrm{GrN}-2031: 10,870 \pm 100, \mathrm{GrN}-2034: 10,590 \pm 100$, all 
Mya phase ages). Alt, in addition to shell date, indicates deposition at very end of Champlain Sea, just before water level regressed to present level of Lake Champlain (29m ASL). The foraminiferan, ostracode and molluscan faunas suggest brackish, subfrigid to cold temperate water conditions.

\section{QC-200. T C 26}

$11,665 \pm 175$

Shell Macoma balthica, Plattsburgh, New York $\left(44^{\circ} 40^{\prime} \mathrm{N}, 73^{\circ} 26^{\prime}\right.$ W), alt 78 to $81 \mathrm{~m}$ ASL. Comment (TMC): oldest date yet obtained for Champlain Sea in USA and indicates deposition early in marine episode. Other US Champlain Sea dates are QG-153: 11,225 \pm 200, Wasner, 1972, W-2311: 11,420 \pm 350, I-3647: 11,230 \pm 170 . These date early stages of sea. Older shell dates from Canada are Gadd et al, 1972, GSC-1533: 12,400 士 160, MacDonald, 1968, GSC-936: 12,000 \pm 200, Lowdon et al, 1967, GSC505: 11,880 \pm 180 . This date, along with 2 others from New York, suggest the following history for sea in Champlain Valley: rapid inundation almost immediately reaching maximum extent, gradual regression due to isostatic crustal rebound, eventual termination of marine episode as connection to marine water source closed. Three dates, all from samples located only a few $\mathrm{km}$ from one another but at different alts, document this history: QC-200: 11,665 \pm 175 , alt 79m ASL, W-1109: 10,560 \pm 350 , alt 55m ASL, QC-199: 10,300 \pm 180, alt 40m ASL.

\section{ARCHAEOLOGIC SAMPLES}

\section{Hudson River series}

Charcoals from Hudson River Archaic sites, subm by L A Brennan, Briarcliff College, Ossining, New York.

QC-111. \#11

$5325 \pm 110$

3375 BC

Firepit \#4, Pear tree loc, Haverstraw quad (41 $11^{\circ} 10^{\prime} \mathrm{N}, 73^{\circ} 52^{\prime} \mathrm{W}$ ), alt ca $12 \mathrm{~m}$, depth ca $90 \mathrm{~cm}$. Coll by LAB. Comment (LAB): dates early use of this loc where middens are extensive (cf, QC-109-110, R, 1976, 18, p 205209).

\section{QC-115. Kemey's Cove}

$4425 \pm 110$

\section{BC}

Hearth, Kemey's Cove, Haverstraw quad (41 $08^{\prime} \mathrm{N}, 73^{\circ} 52^{\prime} \mathrm{W}$ ), alt ca $3 \mathrm{~m}$, depth ca $55 \mathrm{~cm}$. Coll by $\mathrm{R}$ Wingerson. Comment (LAB): sample from salvage dig, hearth was below shell midden, Taconic points were found at this level.

\section{QC-132. Shantok Cove site}

$605 \pm 90$

Charcoal from hearth-pit $\left(41^{\circ} 28^{\prime} 50^{\prime \prime} \mathrm{N}, 72^{\circ} 04^{\prime} 38^{\prime \prime} \mathrm{W}\right)$, alt ca $6 \mathrm{~m}$, depth 51 to $69 \mathrm{~cm}$ below surface. Coll and subm by J Vetter, Dept Anthropol, Adelphi Univ, Garden City, New York. Comment (JV): hearthpit was located in a "living area" adjacent to a small midden. Previous 
dates from site from Isotopes, $845 \pm 125,1035 \pm 150,1110 \pm 95,1190 \pm$ 115 .

\section{Indian Canyon 35WHB(IC-2) series}

Wood charcoal and scorched wood from Indian Canyon, NW Wheeler Co., Oregon ( $\left.44^{\prime} 56^{\prime} 12^{\prime \prime} \mathrm{N}, 120^{\circ} 24^{\prime} 05^{\prime \prime} \mathrm{W}\right)$. Coll and subm by B L Gannon, Portland State Univ, Dept Earth Sci, Portland, Oregon.

General Comment (BLG): samples from fluvial terrace of E-facing canyon cliff. Should date occupational horizon of a "campsite" (food and tool preparation site) as well as truncation of fluvial strata.

QC-135. /re-3

$1460 \pm 100$

Alt ca $7 \mathrm{~m}$, depth $1.1 \mathrm{~m}$. Comment (BLG): sample was expected to be older than IC-2/rc-2 from adjacent occupational area with vague stratigraphic relations dated Gak-3866: $570 \pm 80$.

\section{QC-137. /re-4}

$1545 \pm 100$

Alt ca 6.9 to $7 \mathrm{~m}$, depth 1.6 to $1.75 \mathrm{~m}$. Comment (BLG): age should approx IC-2/rc-3.

\section{Jones Canyon 35WH21(IC-2) series}

Wood choarcoal from Jones Canyon, NW Wheeler Co, Oregon $\left(44^{\circ}\right.$ $\left.55^{\prime} 10^{\prime \prime} \mathrm{N}, 120^{\circ} 14^{\prime} 30^{\prime \prime} \mathrm{W}\right)$. Coll and subm by B L Gannon. Comment (BLG): samples define limits of occupational horizon in House Pits \# 1 and 2 in alluvial fan truncated by a now-epemeral stream.

QC-134. /rc-2 House Pit \#2

AD 1075

$875 \pm 115$

Comment (BLG): previous date from neighboring House Pit \#1 Gak-3867: $360 \pm 80$. Artifact difference suggests different age.

QC-138. /re-3 House Pit \#2

QC-136. /re-4 House Pit \#1

$$
\begin{array}{r}
390 \\
\text { AD } 1560
\end{array}
$$$$
\text { AD } 1650
$$

$300 \pm 75$

$$
2920 \pm 115
$$

970 BC

QC-163. 15

Charcoal from Feature Pit 23 site of Coapexco, Edo de Mexico, Mexico (19 $7^{\prime} 32^{\prime \prime} \mathrm{N}, 98^{\circ} 46^{\prime} 45^{\prime \prime} \mathrm{W}$ ) alt $2600 \mathrm{~m}$, depth $0.85 \mathrm{~m}$ below surface. Coll and subm by $\mathrm{P}$ Tolstoy. Comment (PT): estimated age ca $1150 \mathrm{BC}$, date later within Ixtapalucas phase (1200 BC-950 BC radiocarbon age) than expected. Date acceptable and interpretable as occupation of Coapexco lasted beyond time interval defined, EH-2 (Tolstoy, in press; 1975). EH-2 contemporaneous with Ayotla sub-phase at site AyotlaTlapacoya (Tolstoy \& Paradis, 1970). Samples between 2nd and 3rd trampled floors near F Pit 23 were dated $1180 \pm 160 \mathrm{BC}$ in 1972 within EH-2. 
West Northumberland Canyon series

Charcoal from Triple T Shelter, W Northumberland Canyon, Nye Co, Nevada $\left(39^{\circ} 00^{\prime} \mathrm{N}, 116^{\circ} 55^{\prime} \mathrm{W}\right)$ alt $2073 \mathrm{~m}$. Coll and subm by D H Thomas, Am Mus Nat Hist, Dept Anthropol, New York, New York.

General Comment (DHT): similar silt/living floor sequence as Gatecliff Shelter, central Nevada.

QC-167. 427

AD 1030

$$
920 \pm 105
$$

Unit D5, depth 10 to $20 \mathrm{~cm}$. Comment (DHT): estimated age later than AD 1300, based on cultural assoc.

QC-168. 432

1) $4320 \pm 90$ $2370 \mathrm{BC}$

2) $4880 \pm 120$

Unit C5, depth 170 to $180 \mathrm{~cm}$. Comment (DHT): estimated age older than $1500 \mathrm{BC}$, based on cultural assoc.

QC-169. 415

$5000 \pm 170$

Unit B4, depth 200 to $210 \mathrm{~cm}$. Comment (DHT): estimated age older than 1500 BC, based on cultural assoc.

QC-170. 443

$6340 \pm 160$

Unit B5, depth 360 to $370 \mathrm{~cm}$. Sample diluted with "dead" benzene. Comment (DHT): estimated age should be oldest date in series based on stratigraphy.

QC-171. 422

$3720 \pm 95$

$1770 \mathrm{BC}$

Unit C5, depth 110 to $120 \mathrm{~cm}$. Comment (DHT): estimated age ca $1500 \mathrm{BC}$, based on cultural assoc.

QC-172. 431

1) $2700 \pm 105$

$750 \mathrm{BC}$

Unit C4, depth 100 to $110 \mathrm{~cm}$. Comment (DHT): estimated age $100 \mathrm{BC}$ to AD 500, based on cultural assoc.

\section{QC-173. Calvary Cemetery}

Shell fragments (Ostrea virginica and Venus mercenaria) from Woodside, New York $\left(40^{\circ} 44^{\prime} \mathrm{N}, 74^{\circ} 56^{\prime} \mathrm{W}\right)$. Coll and subm by $\mathrm{S}$ Coughlin, Dept Earth \& Environmental Sci, Queens Coll, Flushing, New York. Comment (SPC): sample possibly from a Colonial period midden exposed during excavation. 


\section{Ostego County series}

Hearth charcoal from Russ site, Ostego Co, New York $\left(42^{\circ} 21^{\prime} 57^{\prime \prime} \mathrm{N}\right.$, $75^{\circ} 15^{\prime} 7^{\prime \prime} \mathrm{W}$ ), alt $319 \mathrm{~m}$, depth $25 \mathrm{~cm}$. Coll and subm by R E Funk, New York State Mus \& Sci Service, Albany, New York.

General Comment (REF): flood plain of upper Susquehanna R, base of plow zone, intrusive into silt.

QC-176. B

$4350 \pm 170$

From Feature 30, Sec Eos39, Loc 2. Comment (REF): estimated age 1800 to 1900 BC, based on assocs on other upper Susquehanna sites.

QC-177. C

$4050 \pm 190$

From Feature 1, Sec E953, $2100 \mathbf{B C}$ zene. Comment (REF): estimated age 3000 to 4000 BC.

\section{Hansanlu Tepe series}

Charcoal from Iron age site $\left(37^{\circ} 01^{\prime} \mathrm{N}, 45^{\circ} 28^{\prime} \mathrm{E}\right)$, Azerbaigan, NW Iran, near town of Nazadeh. Samples from roofbeam of collapsed Iron age buildings of Period IVB. Coll and subm by L Winter, Dept Art, Queens Coll.

QC-183. 18A

Bldg IVE

QC-184. 44A

Bldg IVV

\section{QC-185. 60A}

Bldg V
$2965 \pm 165$

1015 BC

1) $2965 \pm 105$

$1015 \mathrm{BC}$

2) $2950 \pm 90$

1000 BC

Connally, G G and Sirkin, REFERENCES Geol Soc Amer Bull, v 82, p 989-1008.

Elson, J A, 1962, Pleistocene geology between Montreal and Covey Hill, in: Clark, T H (ed), New England Intercollegiate Geol Conf guidebook, 54th Annual Mtg, Montreal, p 61-66. dian Jour Earth Sci, v 6, p 367-372.

Gadd, N R, Lasalle, R, Dionne, J C, Shilts, N W, and MacDonald, B C, 1972, Quaternary geology and geomorphology: Southern Quebec, Montreal, 24th Internatl Gicol Cong livret guide des excursions, A44-C44, $70 \mathrm{p}$.

Hillaire-Marcel, C, 1974, Le deglaciation au nord-ouest de Montreal: Donne radiochronologiques and faits stratigraphiques: Rev Geog Montreal, v 29, p 407-417.

Ives, P C, Levin, B, Robinson, $\mathbf{R}$ D, and Rubin, M, 1964, US Geological Survey radio-
carbon dates VII: Radiocarbon, 6 , p 37-76. 
Lowdon, J A, Fyles, J G, and Blake, W Jr, 1967, Geological Survey of Canada radiocarbon dates VI: Radiocarbon, $v 13$, p 255-324.

MacDonald, B C, 1968, Deglaciation and differential post glacial rebound in the Appalachian region of Southeast Quebec: Jour Geol, v 76, p 664-677.

Pardi, R, 1976, Queens College radiocarbon dates I: Radiocarbon, v 18, p 205-209.

Tolstoy, P, 1975, Settlement and population trends in the basin of Mexico from 1500 B.c. to 650 BC (Ixtapaluca and Zacatenco phases): Jour Field Archaeology, v 2, p 331-349.

in press, The archaeological chronology of Western Mesoamerica before 900 A.D., in: Meighan, C W (ed), Chronologies in New World Archaeology: New York, Seminar Press, $60 \mathrm{p}$.

and Paradis, I, 1970, Early and Middle Preclassic culture in the basin of Mexico: Science, v 167, p 344-351.

Wasner, W P 1972, Ice margins and water levels in northwestern Vermont, in: Doolan, B L and Stanley, R S (eds), New England Intercollegiate Geol guidebook, 64th Annual Meeting, Burlington, p 317-342. 